\title{
Frequency-cascade scheme to the elastic wavefield inversion: A strategy for low wavenumber velocity estimate and several applications
}

\author{
Akio Sakai \\ Japan Petroleum Exploration Co., Ltd. (Japex) \\ 2-2-20 Higashi-shinagawa, Shinagawa-ku, Tokyo 140-0002 Japan, e-mail: akios@japex.co.jp
}

\begin{abstract}
The importance of the elastic wave velocity is known such as in the seismic exploration of gas hydrates, but it has been rarely analysed in practice. While nonlinear elastic wavefield inversion is promising to estimate elastic wave velocity compared with other conventional methods, there has been practical difficulty in searching relatively longer wavelengths by the local iterative methods. To overcome its difficulty, frequency-cascade scheme is examined in space-time domain formulation. Elastic wavefield inversion is conducted under several approaches such that $\mathrm{Vp}$ or Vs is constrained when inverting the other, that $\mathrm{Vp}$ and $\mathrm{Vs}$ is alternately inverted at each step, and that $\mathrm{Vp}$ and Vs are simultaneously inverted. Simultaneous mode proved to be the most effective both in efficiency and accuracy of the estimate. It is proved that frequency-cascade scheme on the simultaneous mode is effective in practice for the models over wide value ranges of elastic wave velocities and Poisson's ratios.
\end{abstract}

\section{Introduction}

Elastic wave velocity is the basic physical quantity for lithology and liquid or solid identification (conventional hydrocarbon, gas hydrates, carbon dioxide, etc.), subbasalt/subsalt imaging, and others.

Standard velocity analysis methods such as stacking velocity analysis, DMO, pre-stack time/depth migration, and others are based on the image enhancing principle for the velocity estimate, and then they have a limited capability for the high resolution and accuracy in velocity estimate. Usually they only estimate compressional wave velocity ( $\mathrm{Vp}$ ). Nonlinear elastic wavefield inversion method is innovative as it provides higher resolution with good accuracy and the full estimation of elastic wave velocity if it works optimally in practice.

Relating to the estimate of gas and gas hydrate quantity, the method has been successfully applied to several data acquired offshore Japan, permafrost in Canada and others on the BSR (Bottom Simulating Reflectors) and gas hydrate bearing zones. It provided the good estimate of velocity and the amount of gas and gas hydrate, and the estimated fine velocity structures became the good constraints to give the speculation of the geological process. These studies, governed by the full elastic wave equations, presumed that there were empirical relations between compressional wave velocity and shear wave velocity (Vs) and medium was horizontally layered, as the subsurface geological structures were moderate. I will briefly summarize the contributions to the gas hydrate studies in Japan by elastic wavefield inversion.

In my recent researches (Sakai, 2002a, 2002b), I evaluated the nonlinear elastic wavefield inversion method by frequency-cascade scheme combined with simultaneous mode for several 2-dimensional geological models to estimate full elastic wave velocity structures. It is the generalization of the inversion formulation by the reflectivity method so far conducted. The following is the summary of the approach to 2$\mathrm{D}$ full elastic wavefield inversion in my recent

\section{$7^{\text {th }}$ International Symposium on Recent Advances in Exploration Geophysics in Kyoto at Kyoto University during January 27-28, 2003}


researches.

- Examine the process of the model update in the viewpoint of the inversion efficiency and stability of the estimates by frequency-cascade scheme in case that the initial model discrepancy is large from the true model. - Evaluate the efficiency of several elastic wavefield inversion modes, i.e., simultaneous mode, alternating mode, and constrained modes.

They were examined for the complex models, say, anticlinal and synclinal structure, lateral velocity varied layered structure, rock intrusion structure, Marmousi model, and so on. For all the models, the proposed approach turned out to be efficient and effective to estimate the elastic wave velocity.

\section{Forward modeling}

Elastic wavefield is governed by linear firstorder coupled equation for particle velocity and stress with isotropic constitutive relation in Cartesian coordinates, and computed by the 2dimensional finite-difference method with second-order accurate time and fourth-order accurate space scheme staggered-grid formulation with optimized coefficients (Levander, 1988, Holberg, 1987). In this study, source and receivers are located in the water and pressure is measured at the receivers in the marine multi-channel reflection survey of conventional and deep-towed seismic systems (For DTAGS survey offshore Japan, refer to Sakai, 1998a, b).

\section{Inversion}

Elastic wavefield inversion scheme is essentially based on the iterative search method of Tarantola (1986) to utilize the local gradients of the misfit function. Misfit function is defined by the least-squared difference functional ( $L 2$ norm) between the observed data and the synthetic data over receivers and shots at all time samples as follows,

$$
E=\sum_{\text {shots }} \int d t \sum_{\text {receivers }}\left|S_{\text {syn }}-S_{\text {obs }}\right|^{2}
$$

where Ssyn is the synthetic wavefield and Sobs is the observed seismic data. A priori data covariance matrix is set unit by normalizing data, and model covariance matrix is set infinite with assuming no a priori information of the model. Inversion is driven to minimize the misfit function in the model space. The gradient of steepest descent $g n$ indicates the direction where the misfit function is reduced most rapidly in the neighbourhood of the starting model. Model vector $m n$ and step length $\alpha n$ at the $n$-th stage is given, and then model vector is updated to reduce the misfit function in step by step line-search mode. Preconditioning by finite a priori model covariance matrix is also examined in both cases of more general non-diagonal and diagonal matrix with conjugate gradient formalism (Mora, 1987).

$$
\begin{aligned}
& m_{n+1}=m_{n}-\alpha_{n} C_{m} g_{n} \\
& E\left(m_{n+1}\right)<E\left(m_{n}\right)
\end{aligned}
$$

The gradient of the misfit function is computed by backpropagating the data residuals and correlating the result with the forwardpropagated wavefields. Update in the model space is done consequently in two stages, firstly Lame coefficients $\lambda$ and $\mu$, and then elastic wave velocities Vp and Vs in the following formulae. 


$$
\begin{aligned}
& \delta \hat{\lambda}_{n}=-\sum_{\text {shots }} \int_{d t}\left(\frac{\partial \overleftarrow{u}_{x}}{\partial x}+\frac{\partial \vec{u}_{z}}{\partial z}\right)\left(\frac{\partial \vec{u}_{x}}{\partial x}+\frac{\partial \vec{u}_{z}}{\partial z}\right) \\
& \delta \hat{\mu}_{n}=-\sum_{\text {shots }} \prod_{d t} 2\left\{\left(\frac{\partial \overleftarrow{u}_{x}}{\partial x} \frac{\partial \vec{u}_{x}}{\partial x}+\frac{\partial \overleftarrow{u}_{z}}{\partial z} \frac{\partial \vec{u}_{z}}{\partial z}\right)+\left(\frac{\partial \overleftarrow{u}_{x}}{\partial z}+\frac{\partial \overleftarrow{u}_{z}}{\partial x}\right)\left(\frac{\partial \vec{u}_{x}}{\partial z}+\frac{\partial \vec{u}_{z}}{\partial x}\right)\right\}
\end{aligned}
$$$$
\delta \hat{\rho}_{n}=-\sum_{\text {shots }} \int d t\left(\overleftarrow{u}_{x} \frac{\partial^{2} \vec{u}_{x}}{\partial t^{2}}+\overleftarrow{u}_{z} \frac{\partial^{2} \vec{u}_{z}}{\partial t^{2}}\right)
$$

$$
\left(\begin{array}{c}
\delta \hat{V}_{p} \\
\delta \hat{V}_{s} \\
\delta \hat{\rho}
\end{array}\right)=\left(\begin{array}{ccc}
2 \rho V_{p} & 0 & 0 \\
-4 \rho V_{s} & 2 \rho V_{s} & 0 \\
V_{p}^{2}-2 V_{s}^{2} & V_{s}^{2} & 1
\end{array}\right)\left(\begin{array}{l}
\delta \hat{\lambda} \\
\delta \hat{\mu} \\
\delta \hat{\rho}
\end{array}\right)
$$

where forward wavefield is illustrated in the forward vector arrow showing forward in time and backward wavefield is in the backward vector arrow showing backward in time. In the similar way, the impedance can be estimated in place of velocity update. For the compressional wave velocity estimate, $\lambda$ will be updated in the iterations and for the shear wave velocity estimate, both $\lambda$ and $\mu$ shall be updated.

There are several strategies of the elastic wave velocity estimate in this scheme according to the real data condition. They are the case that there is good correlation between compressional wave velocity and shear wave velocity and the case that there is no information to establish empirical relations beforehand.

In the latter case, there may be following approaches in real data handling.

1) Vp and Vs are simultaneously updated in the model space (Vp, Vs) assuming no functional relation between $\mathrm{Vp}$ and $\mathrm{Vs}$ in advance,

2) Spatial distribution of $V p$ is known somehow and only Vs is updated in the model space (Vs), 3 ) In case that there is no information of $V p$, update of $\mathrm{Vp}$ alone is done with Vs values fixed in the model space (Vp), and then take the approach 2).

4) In case that no explicit relations between $V p$ and $\mathrm{Vs}$ are known and there is no information of $\mathrm{Vp}$ distribution, update is performed in the alternating mode, i.e., first Vp update with Vs fixed, and then Vs update with $\mathrm{Vp}$ fixed in the model space (Vp, Vs) at each iteration step.

I tentatively call them; simultaneous mode (case 1), two-stage constrained mode (cases 2 and 3), alternating mode (case 4). The approach by acoustic inversion is not categorized by the above modes, but it has a limited functionality of velocity update in model space $(\mathrm{Vp})$ by its approximated governing equation.

Inversion trial for these modes in case of no empirical relations between $\mathrm{Vp}$ and $\mathrm{Vs}$ demonstrated that simultaneous mode has the highest efficiency and accuracy of the final model. Alternating mode turned inefficient in the present models, which were thought to be in practice.

Because of low sensitivity of density in inversion and to concentrate on the velocity effect, density $\rho$ is varied with compressional wave velocity under the assumed empirical relation in the current study.

\section{Model update}

In model updates, they converge to the local minima close to the starting point in the model space. Several attempts have been done to handle local minima after the nonlinear wavefield inversion methods appeared in the publications. If there is a priori constraints 
such as well data, traveltime analysis, etc., it shall be taken into account to construct starting models. Starting from different models is one way to judge if it is global minimum, but it is not confirmed if they do reach the real minimum among local minima. Global searching methods such as Monte Carlo method would be impractical, as it is computerintensive task now.

In the current study, I took the following modified multi-scale approach.

1) Start simplest models (e.g., Vp and Vs linearly increasing in depth).

2) Choose lowest dominant frequency Fmin in the source spectrum band and widest offset distance range

3) Increase dominant frequency at the rate, say, by two times and fix the offset distance range (frequency-cascade scheme)

4) Decrease the maximum offset

By this scheme, spatial frequency velocity bands get broader, and the model velocity structure is made finer. Nearest traces contribute to finer compressional wave velocity distribution once the lower wavelength trend is acquired. The frequency-cascade scheme is illustrated schematically in Figure 1. Starting from the lower frequency (frequency 1), the minimum trough width including the global minimum is relatively large, and then updated models will not be trapped in local minima even starting from the models showing large departure from the true model. With approaching the minimum, we transitionally shift to the next higher frequency (frequency 2) where the next starting point is in the minimum trough width range in the higher frequency (See Fig.1). The number of iterations is mitigated in each cascade frequency with quality check of iteration process and the total computation time is comparable with or better than the case of a single broad source function.

If the multigrid scheme is properly incorporated, the computation efficiency can be extremely enhanced. Starting from coarser grid size and long time sampling rate and decreasing the grid size and time sampling rate, the estimated velocity model is made finer (multigrid scheme). For real data handling, the depth or time range shall be windowed with combining above steps with model-dependent ideas (space and time cascade scheme). Multiscale approach in case of the acoustic inversion was discussed in Bunks et al., (1995). Criterion how to choose the frequency and offset was discussed by Sirgue and Pratt (2001) for an acoustic model with a single deeper target under the frequency domain inversion formulation. I picked the dominant frequency of synthetic gaussian or ricker wavelet source in the simulation studies, and as the frequency spectra were shifted with overlapping, there was no similarity with sinusoidal frequency cascading by them. It was the process of broadening the source spectral band in practice. To compare with each other, sinusoidal wavetrains were also examined, which needed more iteration number with more frequencies.

\section{Benefits of the elastic wave velocity inversion to several problems}

There are much benefits of the elastic wavefield inversion over acoustic inversion. Such examples are shown as follows.

- Lithology identification

- Liquid or solid identification

- Below gas cloud imaging

- Subsalt and subbasalt imaging 
If multi-component elastic inversion and anisotropic constitutive relation is incorporated into implementation, it may be used for the identification of fracture orientation. Anyway acoustic wave inversion by an acoustic equation is not sufficient and makes use of part of the full inversion functionality. It can be only applied to a fluid medium.

Another good example of the application is a monitoring of the carbon dioxide injection related to EOR or sequestration program. The state of injected carbon dioxide was well constrained by Poisson's ratio estimate with pore pressure change in time-lapse observations (e.g., Gritto et al., 2002).

There are many examples in the hydrocarbon exploration, but in almost all cases analyses are done consecutively, that is, firstly compressional wave velocity estimate by conventional velocity analysis method with a limited resolution, and then stratigraphic and a sort of AVO techniques with logging data constraints. This is a sort of 'constrained mode' in my context. But it is absolutely not confirmed that such step-wise process reaches reality with efficiency and accuracy.

\section{Gas hydrate researches for the applications of elastic wave velocity estimate}

Gas hydrate is the clathrate compound of host water with guest gases of lower molecular weight such as methane, and stable under the higher pressure and lower temperature conditions, while in the marine subsurface conditions the water is deeper and the gas hydrate in sediment is shallower in depth below seabed.
To form hydrates, the following three conditions are necessary.

- Adequate gas molecules to stabilize most of hydrate cavities

- Sufficient water molecules to form the cavity

- Temperature and pressure within the hydrate equilibrium region

As the subsurface temperature below seabed raises with the geothermal gradient (3-4 $\operatorname{deg} \mathrm{C} / 100 \mathrm{~m}$ in the Nankai trough), natural gas hydrates are stable above the boundary, called gas hydrate stability zone (GHSZ), below which free gas is expected to be as the second phase. The phase boundary is observed and visualized as BSR in zero-offset seismic section in reversed polarity of the seabed reflection due to the acoustic impedance difference between natural gas hydrate that shows larger acoustic impedance and free gas underneath that shows smaller acoustic impedance. The distribution of the amount of these quantities will be quantitatively estimated by the elastic wave velocity.

\section{Hybrid surveys with frequency-cascade scheme and multicomponent concept}

Gas hydrate exploration is important both in environmental and future natural resource mitigation problems. BSR is believed to be the main key indication for the existence of gas hydrates and related gas.

BSRs were observed broadly in the deep marine area around Japan by several seismic surveys since early 1970s. After reviewing and examining the specifications of data acquisitions conducted in the past, a couple of experimental seismic surveys were executed in the eastern Nankai trough during the period of 1995 to 2000 as part of $R \& D$ program of 
Methane Hydrates Consortium of 11 organizations in Japan (see in detail, Annual Methane Hydrates R\&D Reports 1995-2000; Arai, Y. \& Ohara, T. as the project managers and editors of the report, Sakai, A. as the chief researcher and author of the report of the Branch of Geophysical Disciplines in them). It was named 'Hybrid survey". These surveys were intended to complement denser line grid survey conducted there by MITI for the determination of the drilling location in 1999-2000. The main objective of the experiment was to design and evaluate the optimal data acquisition specifications, and these data was utilized for defining fine subsurface structures related to BSR and hydrate quantification based on the rock physics models.

In these surveys, frequency bands range from approx. 5 to $650 \mathrm{~Hz}$ as the combination of several survey specifications in the frequencycascade scheme, minimum CMP interval is $3.125 \mathrm{~m}$ and maximum offset exceeds $10 \mathrm{~km}$ for ocean-bottom seismograph surveys. By multi-component ocean-bottom seismograph deployment, elastic wave velocity structure was evaluated by identifying converted shear waves above and below BSR by tomographic method. It was the first case in the world to analyse velocity based on CMP for multichannel deeply towed survey (DTAGS collaborated with Naval Research Laboratory, USA). Though DTAGS was conducted some times so far, velocity analyses had not been based on CMP gathers due to lack of reliable navigation data or uncorrected positioning data. The higher frequency bands (DTAGS in $250-650 \mathrm{~Hz}$ ) seismic data was expected to provide insight on frequency dependency of the seismic waves and hopefully fill the frequency gap to the higher frequency data of laboratory measurements. The frequency-cascade scheme concept I proposed in the inversion had been realized in the multiple surveys of overlapping incremental dominant frequency bands, not limited to the current inversion concept at that time, to enhance resolution.

By imaging reflectors and analysing velocities, the relation between the velocities and geological structures such as faults, lapping on unconformity, and the relation between seismic amplitudes and velocity structures, and others could be discussed. The latter was related with the phenomena of "blanking" and which has been a long-standing problem. From all of the aspects, the experiments conducted offshore Japan in this period was prominent and promising for the marine BSR and gas hydrate research (Sakai, 1998a, 1998b).

\section{Correction of missing sonic log data and Double BSR}

After 1999 MITI drilling campaign, results and several problems were discussed at WPGM (Western Pacific Geophysical Meeting of AGU) at Tokyo in June 27-29, 2000.

Densely traversed high resolution 2dimensional seismic survey data were reconstructed to 3-dimensional data volume after adjusting intersection errors along the traversed lines, applying matched filter on these points, and fitting optimally curved reflection surfaces for trace interpolation. Guided by this quasi-3D volume with $2 \mathrm{D}$ higher resolution image and VSP data of the wells, BSRs and velocity structures were mapped with accuracy. Lower velocity zone between BSRs and higher velocity bounds demonstrated by VSP analyses may indicate the existence of gas accumulation 
in the pore space close to drilled well positions.

Hydrate existence zones were mapped above BSRs and hydrate free zone exists between mapped hydrate zone and BSR. This gap is represented by relatively low velocity. The reason why such gapped zone exists is not obvious. One possible explanation would be new BSR appearing in the depth shallower than relic one of stronger amplitudes while prominent spatial continuation of the horizon were not identified as intersections with normal sediment stratification in seismic sections.

Two strong amplitude reflectors were noticed, which corresponded to two highly attenuated zones over these wells. The existence of such double BSR posed the question in the eastern Nankai trough. Dependent on the specific field (Cascadia margin, North Sea and others), different origins might be. In this study, although borehole sonic logging data (DSI) did not succeed in providing reliable estimate due to high attenuation, wavefield inversion was successfully applied to estimate the compressional wave velocity including the sonic missing zone due to high attenuation for zero-offset VSP data (Sakai, 2000).

It is essential to estimate the reliable elastic wave velocity for further study especially in case of no logging data.

\section{Gas hydrate saturation model:}

\section{Compaction model or Cementation model?}

In gas hydrate exploration, shear wave velocity is essential in the problem how to determine the gas hydrate saturation model, one extreme model is compaction model which is characterized by gas hydrates disseminated in the pore space and no conspicuous hydrate cementation with grains, and the other extreme model is cementation model which is characterized by gas hydrate cemented at the grain contacts or on the grains. The estimate of the amount of gas hydrate by elastic wave velocity is strictly dependent on the models.

\section{In JAPEX/JNOC/GSC Mallik 2L-38,} Mackenzie delta, NWT, Canada, it was discovered that the elastic wave velocity of gas hydrates was well simulated by means of compaction model for calibrated elastic wave velocity data (Sakai, 1999). Prior to this analysis, well-calibrated full elastic wave velocity data had not been in use to fix a model. In the subsequent researches on the problem of the gas hydrate place in pore, it was proved that compaction model was the standard model to estimate the amount of gas hydrate based on the elastic wave velocity. It was also affirmed by MITI Nankai trough research wells in the eastern Nankai trough (Sakai, 2000). In these researches, seismic and logging data were utilized and the seismic methods turned out to be useful for the estimate of the amount of gas hydrates with reliable elastic wave velocity estimates.

Such examples are illustrated in Fig. 2. In the gas hydrate highly saturated depth zone of Nankai trough wells, compaction models were successfully applied to the DSI data, which was calibrated and supplemented by VSPs. With evaluated lithology (sand/shale and critical porosity) and porosity values, the calibrated DSI data well fitted the synthetic model data under the gas hydrate saturation estimated by Archie's formula on resistivity data. Uncertainties of well data were also examined in the course of model parameter evaluation. 
Simulation examples of frequency-cascade scheme and linear starting models

If the subsurface geological structures are moderate, wavefield inversion by the reflectivity method is efficient in the estimate of 1-D wave velocity with attenuation quality factor for problems adapted for survey geometry and data quality in either domain of $\tau-p, t$-depth, $t$-offset distance, and frequency with - $p$, -depth, or -offset distance. In many cases, shear wave velocity was constrained by compressional wave velocity in these studies for simplicity's sake. It is a powerful and flexible method while it is restricted to the 1-D condition. In order to alleviate these conditions, finite-difference modeling and full elastic wavefield inversion by simultaneous mode and others was examined recently as summarized in the previous sections.

Here I illustrate an example of the applications of the proposed method.

A model is an intrusion of 2-D rock mass characterized by layers of lithology change and gas saturation, which is placed in a laterally invariant linearly downward increasing background $\mathrm{Vp}$ and $\mathrm{Vs}$ field with a linear relation between Vp and Vs. I examined several models of the similar shape with changing linear relations of $\mathrm{Vp}$ and $\mathrm{Vs}$ and $\mathrm{Vp}$ values. A background sediment layer is taken as a starting initial model of inversion. Shot interval, receiver interval, grid size and sampling rate are $200 \mathrm{~m}, 100 \mathrm{~m}, 20 \mathrm{~m}$, and $2 \mathrm{msec}$. Maximum initial velocity perturbation is approx. $10 \%$ in $\mathrm{Vp}$ and $20 \%$ in Vs relative to the true model. The inverted model velocity structure is less than $2 \%$ relative error at most (see Figs. 3$6)$.
Vp vs. Vs relations are key indication to determine lithology as well as for direct seismic identifications of pore fluid using, say conventional AVO analysis. I take as an example a 1-D model of another linear relation of $\mathrm{Vp}$ and $\mathrm{Vs}$ and display starting model, true model and final inverted model in Vs vs. Vp/Vs diagram for it. Starting model is simply shown as a quasiconstant $\mathrm{Vp} / \mathrm{Vs}$ line with $\mathrm{Vp}$, but after inversion, the lithology and state unit is clearly separated into pieces in this diagram with accuracy (see Fig. 7).

A starting model of linearly downward increasing background $\mathrm{Vp}$ and $\mathrm{Vs}$ illustrates the ignorance of the prior knowledge of the elastic velocity structure, while it is taken to implicitly introduce turning waves for background reflectors to stabilize the low wavenumber velocity inversion. It worked well in the same manner as a deeper background velocity layer of a simple acoustic model of Mora (1989). It is shown that it provides a better model if combined with frequency-cascade scheme.

\section{Discussion and conclusions}

1. There are many benefits to use information of the elastic wave velocity. Elastic wavefield inversion provides most promising approach to estimate it with good accuracy and resolution.

2. Gas hydrate estimate by the elastic wave velocity is one of the effective and practical applications. Major work has been done under the constrained approach. It was shortly reviewed with concentrating on my studies. The current approach proves to be of use by experimental studies.

3. The selection of the initial starting model is 
the most important in the nonlinear wavefield inversion method if it uses the local line searching method with gradients as in my studies. Global searching method is still out of the current computational power, and I examined to use the frequency-cascade scheme, i.e., starting from the lowest dominant frequency and increasing at the rate of two time or close to it, which can be combined with the selection of the offset ranges.

\section{One promising method of initial model} selection is to choose a linearly increasing velocity model of $\mathrm{Vp}$ and Vs combined with the model update by frequency-cascade scheme. It implicitly includes turning waves, and corresponds to the deepest reflected waves advocated in acoustic inversion by Mora (1989) to stabilize low wavenumber background velocity inversion.

5. In the elastic wavefield inversion, there are several approaches for physical parameter estimate. In many cases, we can assume the existence of the empirical relation between $\mathrm{Vp}$ and Vs. In such cases, Vs values are constrained by $\mathrm{Vp}$ values and it provides the efficient inversion scheme. If there are no such relations, there are alternating mode, simultaneous mode and others. For model simulation studies, the simultaneous mode proves to be the most efficient and stable in the final model estimate. In real data handling, there used to be the case that taking two-stage estimate, that is, firstly Vp and then Vs. It is the scheme that the analysis is done firstly using acoustic analysis and/or inversion. Simulation studies showed that simultaneous mode was most efficient and workable approach.

\section{There are discussions between two}

approaches of frequency domain and space-time domain inversion on its feasibility, efficiency and others. Frequency domain approach has been advocated having the advantage on the computational efficiency in the regular geometry. The space-time domain approach has strong benefits in the flexibility of inversion by setting the optimized window of the shot data in the space-time domain. Such requirements quite often occur in the real data inversion, which can be called 'space and time cascade scheme'. The frequency domain approach requires the excess memory and partly because of it, I guess, almost all inversion of real data would have been restricted to acoustic inversion in frequency domain method. The computational efficiency in space-time domain inversion is to be overcome by parallel computation for forward modeling, while it is beyond the current studies.

7. By the current simulation study, elastic wavefield inversion approach requires the data acquisition specifications in the marine multichannel survey to take as low frequency as possible and as long a maximum offset as possible to estimate lower wavelength velocity trend in itself. Trial as a frequency-cascade scheme so far conducted in gas hydrate seismic surveys in Japan is useful for such unified analysis by the elastic wavefield inversion approach.

\section{References}

Bunks, C., Saleck, F.M., Zaleski, S., and Chavent, G., 1995, Multiscale seismic waveform inversion, Geophysics, 60, 1995

Gritto, R.M., Daley, T.M., and Myer, L.R. 2002, Joint cross well and single well seismic studies 
of $\mathrm{CO} 2$ injection in an oil reservoir, preprint

Holberg, O., 1987, Computational aspects of the choice of operator and sampling interval for numerical differentiation in large-scale simulation of wave phenomena, Geophys. Prosp., 35, 1987

Ji, Y., Sakai, A., and Singh, S., 2000, Waveform inversion of vertical seismic profile, EAGE, 2000

Levander, A., 1988, Fourth-order finitedifference P-SV seismograms, Geophysics, 53, 1988

Mora, P., 1987, Non-linear two-dimensional elastic inversion of multi-offset seismic data, Geophysics, 53, 1987

Mora, P., 1989, Inversion = migration + tomography, Geophysics, 54, 1989

Sakai, A., 1998a, Broad-band seismic data acquisitions for fine scale imaging and velocity determination associated with BSR, European Geophysical Society 1998

Sakai, A., 1998b, Seismic studies related to gas hydrate in the Nankai Trough, JNOC International Methane Hydrate Symposium 1998

Sakai, A., 1999, Velocity analysis of vertical seismic profile (VSP) survey at JAPEX/JNOC/GSC Mallik 2L-38 gas hydrate research well, and related problems for estimating gas hydrate concentration, GSC Bulletin 544, 1999

Sakai, A., 2000, Seismic studies by 2D high resolution and reconstructed quasi-3D data combined with VSPs in the eastern Nankai Trough, AGU Western Pacific Geophysics meeting 2000

Sakai, A., 2002, Elastic wave velocity estimate without pre-processing, Technical meeting of the Japanese Association for Petroleum Technology, May 2002 (Abstract in Japanese)

Sakai, A., 2002, Velocity estimates by the nonlinear elastic wavefield inversion method, Proceedings of the 107th SEGJ (the Society of Exploration Geophysicists of Japan)

Conference, Oct. 2002

Sirgue, L. and Pratt, R.G., 2001, Frequency domain waveform inversion: A strategy for choosing frequencies, EAGE, 2001

Tarantola, A., 1986, A strategy for nonlinear elastic inversion of seismic reflection data, Geophysics, 51, 1986

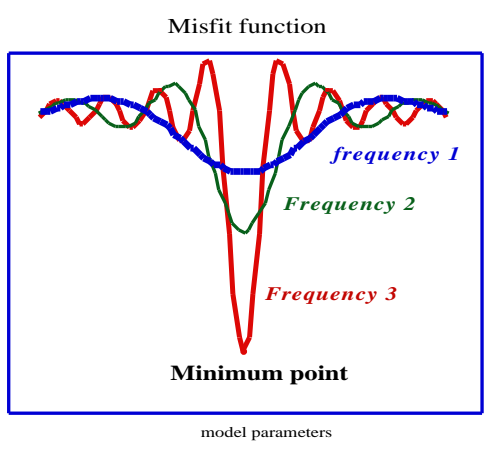

Figure 1 Schematic diagram of frequency-cascade scheme in the model space close to the global minimum point. Each of misfit functions is determined in each of frequencies; narrower valley of the misfit function is for higher dominant frequencies. 

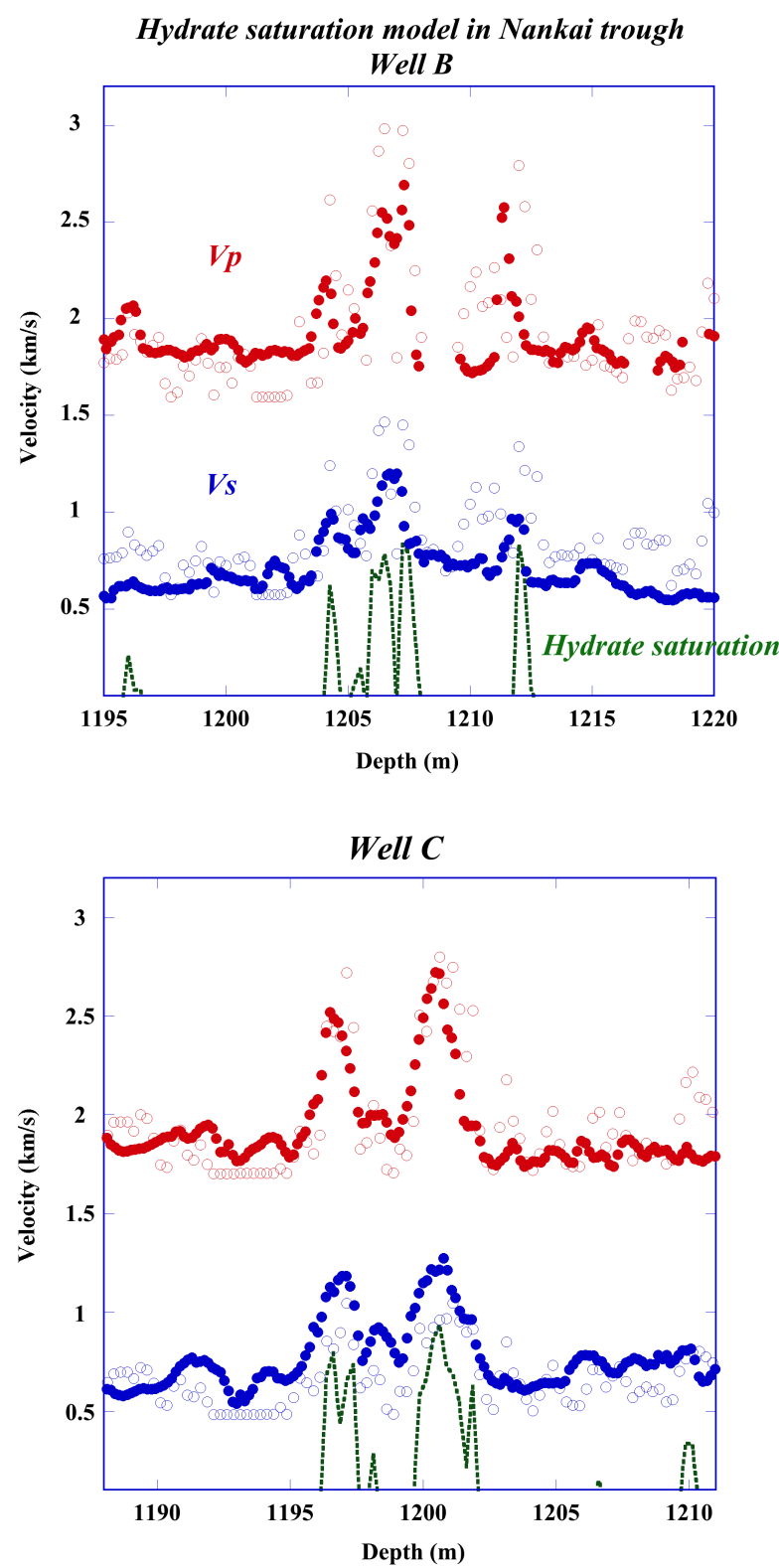

Figure 2 DSI log calibrated by VSPs are correlated by synthetic rock physics model (compaction model). Upper figure is for well $\mathrm{B}$ and lower figure is for well $\mathrm{C}$ in the Nankai trough. Dots of open circle compose synthetic model and dots of filled circle compose calibrated DSI $\log$. Blue dots are shear wave velocity and red dots are compressional wave velocity. Green dot lines illustrate gas hydrate saturation estimated.

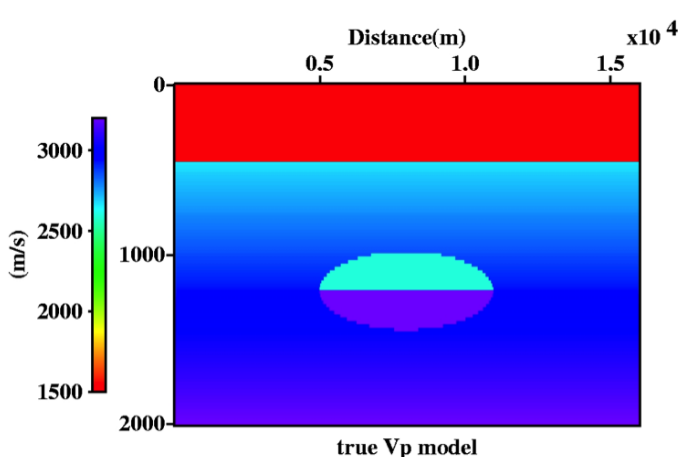

Figure $32-\mathrm{D}$ Vp structure of an intrusion model where the initial model is defined as a laterally invariant linearly downward increasing background $\mathrm{Vp}$ field. (20m final grid size, $2 \mathrm{msec}$ time sampling rate, $200 \mathrm{~m}$ shot interval and $100 \mathrm{~m}$ receiver interval)

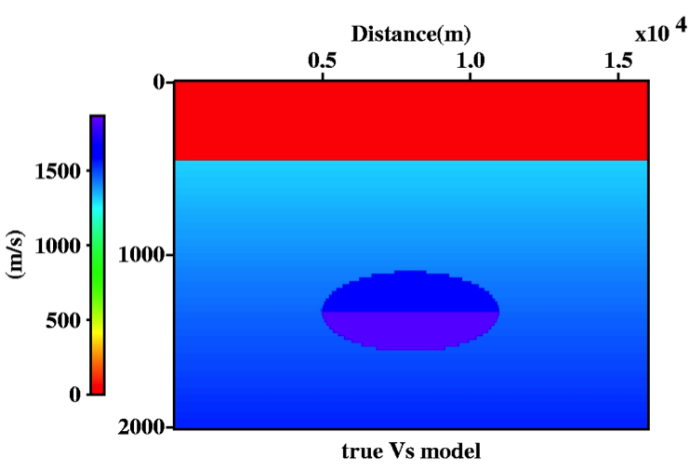

Figure 4 Vs structure of an intrusion model where the initial model is defined as a laterally invariant linearly downward increasing background Vs field

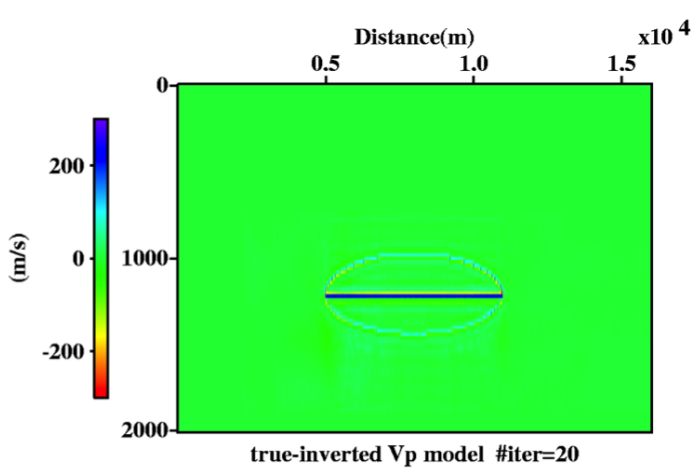

Figure 5 Residual Vp field by frequency-cascade scheme and simultaneous mode inversion 


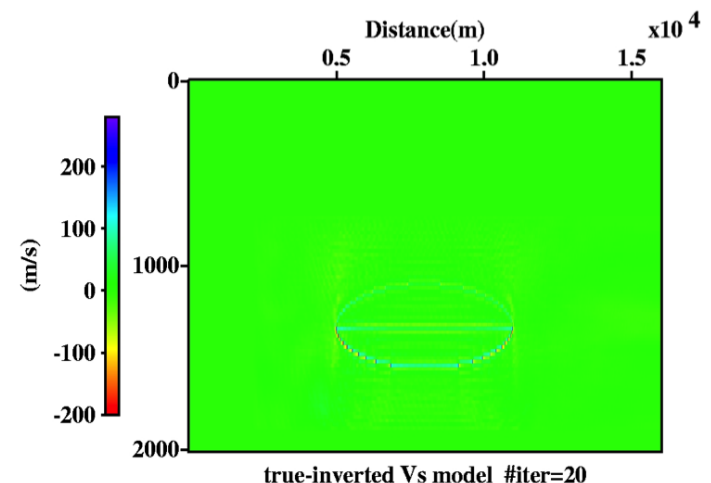

Figure 6 Residual Vs field by frequency-cascade scheme and simultaneous mode inversion
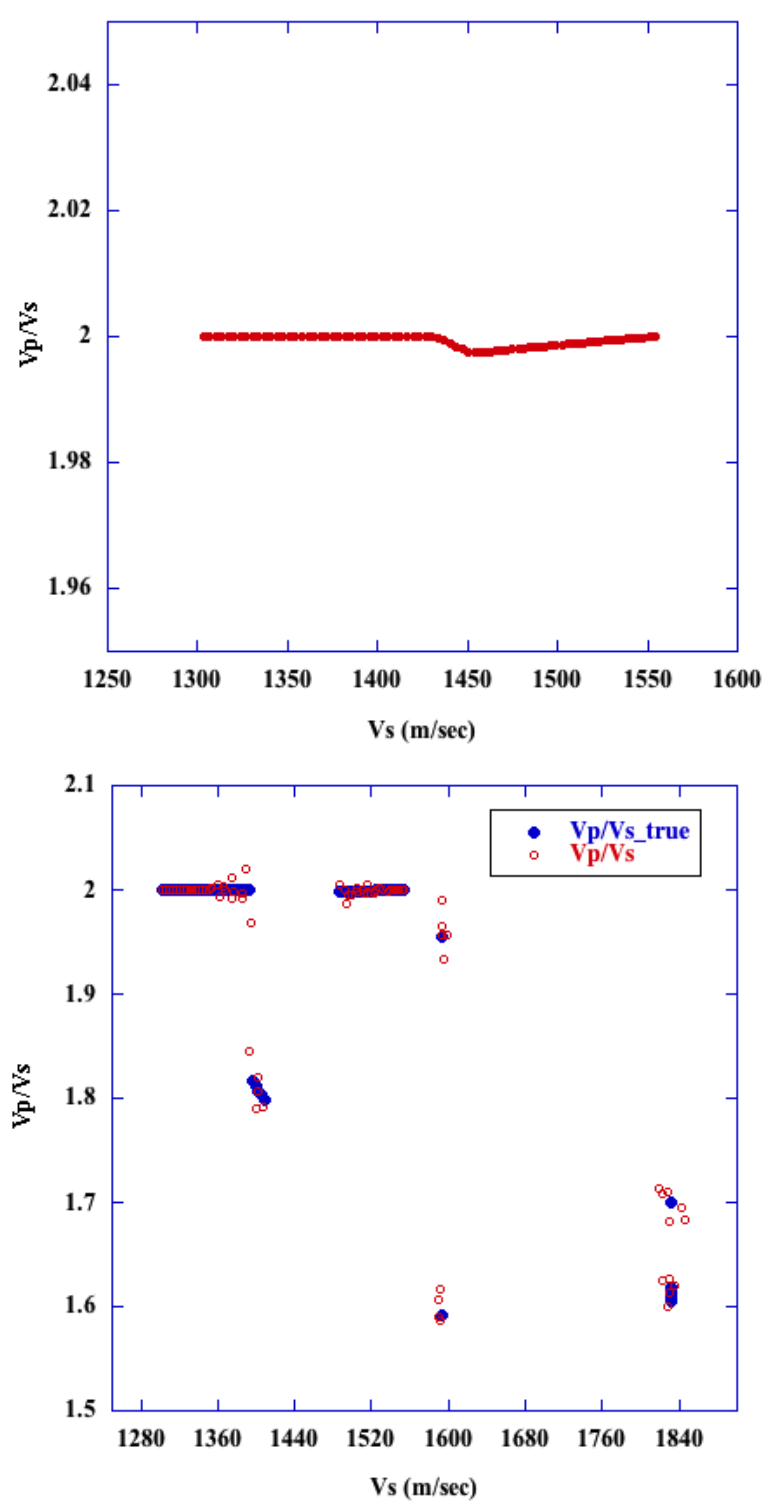

Figure 7 Vs vs. Vp/Vs relations of the starting model (upper figure) and true and inverted model (lower figure) 